\title{
ACTION ON GRASSMANNIANS ASSOCIATED WITH COMMUTATIVE SEMISIMPLE ALGEBRAS
}

\author{
DAE SAN KIM AND PATRICK RABAU
}

\begin{abstract}
Let $A$ be a finite-dimensional commutative semisimple algebra over a field $k$ and let $V$ be a finitely generated faithful $A$-module. We study the action of the general linear group $\mathrm{GL}_{A}(V)$ on the set of all $k$-subspaces of $V$ and show that, if the field $k$ is infinite, there are infinitely many orbits as soon as $A$ has dimension at least four. If $A$ has dimension two or three, the number of orbits is finite and independent of the field; in each such case we completely classify the orbits by means of a certain number of integer parameters and determine the structure of the quotient poset obtained from the action of $\mathrm{GL}_{A}(V)$ on the poset of $k$-subspaces of $V$.
\end{abstract}

\section{INTRODUCTION}

In [4] the second author examined the action on Grassmannians of a general linear group defined over an extension field of the base field. In the present paper we replace the extension field by a finite-dimensional commutative semisimple algebra with several simple components, i.e., we consider the action of products of general linear groups. Let us mention that corresponding situations can be investigated for other classical groups, for instance for symplectic groups [3].

We now present our problem in more detail. Let $A$ be a finite-dimensional commutative semisimple algebra over a field $k$ and let $V$ be a finitely generated $A$-module. We let $\left[{ }^{V}\right]_{k}$, denote the set of $k$-subspaces of $V$, partially ordered by inclusion, and consider the natural action of the group $\mathrm{GL}_{A}(V)$ of $A$-linear automorphisms of $V$ on $\left[\begin{array}{l}V \\ *\end{array}\right]_{k}$. Our main result (Theorem 3.1) is, if $k$ is an infinite field and $V$ is a faithful $A$-module, a necessary and sufficient condition for the quotient poset $\left.\left[\begin{array}{l}V \\ *\end{array}\right]\right]_{k} / \mathrm{GL}_{A}(V)$ to be finite is that $\operatorname{dim}_{k} A \leq 3$. Apart from the trivial case $A=k$, this condition covers five possibilities: $A=F$, $L, k \times k, k \times k \times k$, and $F \times k$, where $F$ and $L$ are respectively a quadratic and a cubic extension field of $k$. The cases $A=F$ or $L$ were treated in [4] and we treat the remaining ones here. In each individual case we show that the quotient poset itself is independent of the particular fields chosen (with one exception for the case $A=k \times k \times k$ and $k$ is the field with two elements). In group theoretic language, we determine the finiteness of the double coset spaces

Received by the editors December 15, 1989.

1980 Mathematics Subject Classification (1985 Revision). Primary 15A03; Secondary 06A99. 
$P \backslash \mathrm{GL}_{k}(V) / \mathrm{GL}_{A}(V)$ with $P$ a maximal parabolic subgroup of $\mathrm{GL}_{k}(V)$ when $\operatorname{dim}_{k} A \leq 3$ and get bounds on their cardinalities.

The following table gives, for all $A$ with $\operatorname{dim}_{k} A \leq 3$, the number of parameters necessary to describe the quotient poset $\left[\begin{array}{l}V \\ *\end{array}\right]_{k} / \mathrm{GL}_{A}(V)$, as well as its asymptotic size for the case of $V$ a free $A$-module of rank $n$.

\begin{tabular}{c|c|c}
$A$ & \# of parameters & asympt. size of poset \\
\hline$k$ & 1 & $n$ \\
$F$ & 2 & $(1 / 2) n^{2}$ \\
$k \times k$ & 3 & $(1 / 3) n^{3}$ \\
$L$ & 4 & $(1 / 48) n^{4}$ \\
$F \times k$ & 6 & $(17 / 5760) n^{6}$ \\
$k \times k \times k$ & 8 & $(11 / 26880) n^{8}$
\end{tabular}

When $k$ is a finite field, one can also compute the size of each orbit for the action of $\mathrm{GL}_{A}(V)$ on $\left[\begin{array}{l}V \\ *\end{array}\right]_{k}$ and obtain as a consequence some new $q$-series identities. This was done in [4] in the context of cubic field extensions, where the author obtained new $q$-analogues of transformation and evaluation formulas for hypergeometric series due to Gessel and Stanton [2]. We will postpone these combinatorial applications in our situation to a later paper.

We should also point out that this work has an important application to the theory of automorphic forms (see for example [1]). Indeed, whenever one has an embedding $G \rightarrow G^{\prime}$ of reductive groups over a global field, one can consider the Rankin integral of cuspforms of $G$ against the pullback of an Eisenstein series on $G^{\prime}$ and hope to get an integral representation of some $L$-functions associated with those cuspforms. This naturally leads to a consideration of double cosets $P^{\prime} \backslash G^{\prime} / G$ with $P^{\prime}$ parabolic subgroup of $G^{\prime}$, which is the subject of the present paper.

The paper is organized as follows. After some preliminaries in $\S 2$, we state the main problem in $\S 3$. $\S \S 4$ to 6 are respectively devoted to the cases $A=$ $k \times k, A=k \times k \times k$, and $A=F \times k$.

\section{Preliminaries}

We will use the following general notation. The group of units of a ring $R$ is written $R^{\times}$. The set of submodules of an $R$-module $U$ is written $\left[\begin{array}{l}U \\ { }_{*}\end{array}\right]_{R}$, and the submodule of $U$ generated by a set $S$ is written $R S$, or $\left\langle v_{1}, \ldots, v_{n}\right\rangle_{R}$ if $S=\left\{v_{1}, \ldots, v_{n}\right\}$. If $R$ is a field, we write $\left[\begin{array}{c}U \\ i\end{array}\right]_{R}$ for the set of subspaces of $U$ of dimension $i$ over $R$. Finally, $\mathbb{N}$ is the set of nonnegative integers, and the set-theoretic difference of two sets $S$ and $T$ will be written $S-T$.

Let $k$ be a field. In this section, we recall and state (mostly without proofs) facts about commutative artinian semisimple algebras over $k$ and modules over them, introducing several definitions and notations along the way. Any such algebra is isomorphic to an algebra $A=k_{1} \times \cdots \times k_{m}$ for some integer $m$, where each $k_{i}$ is an extension field of $k$. The primitive idempotents of $A$ 
are the elements $e_{i}=(0, \ldots, 0,1,0, \ldots, 0)$ with the 1 in the $i$ th position $(i=1, \ldots, m)$; they satisfy $e_{1}+\cdots+e_{m}=1, e_{i}^{2}=e_{i}$, and $e_{i} e_{j}=0$ for $i \neq j$. This notation will remain fixed throughout the paper.

Let $V$ be a module over $A$, which can also be viewed as a vector space over $k$ by restriction of scalars. Each $e_{i} V$ is a submodule of $V$ and $V=$ $e_{1} V \oplus \cdots \oplus e_{m} V$; moreover, each $e_{i} V$ may be viewed as a vector space over $k_{i} \cong A / \operatorname{ann}\left(e_{i}\right) \cong e_{i} A$. If $V^{\prime}$ is another $A$-module, any $T \in \operatorname{Hom}_{A}\left(V, V^{\prime}\right)$ is completely determined by its restrictions $\left.T\right|_{e_{i} V} \in \operatorname{Hom}_{k_{i}}\left(e_{i} V, e_{i} V^{\prime}\right)(i=$ $1, \ldots, m)$. Thus, if $R$-mod denotes the category of (left) $R$-modules, the categories $A$-mod and $k_{1}-\bmod \times \cdots \times k_{m}-\bmod$ are equivalent. This equivalence of categories is the key to many of the results listed below.

Define the dimension vector of $V$ to be the $m$-tuple

$$
\operatorname{dim}_{A} V=\left(\operatorname{dim}_{k_{1}} e_{1} V, \ldots, \operatorname{dim}_{k_{m}} e_{m} V\right) .
$$

In case $m=2$ or 3 , we will talk about the bidimension or tridimension of $V$. Also, call $\operatorname{dim}_{A} V$ finite when all its components are finite.

Lemma 2.1. (a) Two A-modules are isomorphic if and only if they have the same dimension vector.

(b) $\operatorname{End}_{A}(V) \cong \prod_{i=1}^{n} \operatorname{End}_{k_{i}}\left(e_{i} V\right)$ as k-algebras; $\mathrm{GL}_{A}(V) \cong \prod_{i=1}^{m} \mathrm{GL}_{k_{i}}\left(e_{i} V\right)$ as groups. If $\operatorname{dim}_{A} V=\left(n_{1}, \ldots, n_{m}\right)$ is finite, then $\mathrm{GL}_{A}(V) \cong \prod_{i=1}^{m} \mathrm{GL}\left(n_{i}, k_{i}\right)$.

(c) Let $V_{1}$ and $V_{2}$ be submodules of $V$. Then

(i) $\operatorname{dim}_{A} V=\operatorname{dim}_{A} V_{1}+\operatorname{dim}_{A} V / V_{1}$;

(ii) $\operatorname{dim}_{A}\left(V_{1}+V_{2}\right)+\operatorname{dim}_{A}\left(V_{1} \cap V_{2}\right)=\operatorname{dim}_{A} V_{1}+\operatorname{dim}_{A} V_{2}$;

(iii) $\operatorname{dim}_{A} V=\operatorname{dim}_{A} V_{1}+\operatorname{dim}_{A} V_{2}$ if $V=V_{1} \oplus V_{2}$.

(All the sums are componentwise.)

Proposition 2.2. Let $V$ be an A-module.

(a) $V$ is free if and only if $\operatorname{dim}_{k_{1}} e_{1} V=\cdots=\operatorname{dim}_{k_{m}} e_{m} V$.

(b) $V$ is a finitely generated $A$-module if and only if $\operatorname{dim}_{A} V$ is finite. If each $k_{i}$ is of finite degree over $k$, then $V$ is finitely generated if and only if $\operatorname{dim}_{k} V<\infty$.

Here we give more terminology. Let $W$ be a $k$-subspace of $V$. We write $\left[\begin{array}{c}W \\ *\end{array}\right]_{A}$ for the set of $A$-submodules of $V$ contained in $W$. For $k$-subspaces $W_{1}, \ldots, W_{r}$ of $V$, write

$$
W=W_{1} \oplus_{A} \cdots \oplus_{A} W_{r}
$$

if $W=\bigoplus_{i} W_{i}$ and $A W=\bigoplus_{i} A W_{i}$, in which case we say that the sum $\bigoplus_{i} W_{i}$ is a direct sum over $A$. For two summands, we have the equivalent formulation: $W=W_{1} \oplus_{A} W_{2}$ if and only if $W=W_{1} \oplus W_{2}$ and $A W_{1} \cap A W_{2}=0$. The largest $A$-submodule of $V$ contained in $W$ will be called the $A$-component of $W$, written $\operatorname{comp}_{A} W$. For a vector $v \in V$, we have

$$
v \in \operatorname{comp}_{A} W \Leftrightarrow A v \subseteq W .
$$


Lemma 2.3. Let $W_{1}, W_{2} \in\left[\begin{array}{l}V \\ *\end{array}\right]_{k}$. Then

(i) $A\left(W_{1}+W_{2}\right)=A W_{1}+A W_{2}$;

(ii) $\operatorname{comp}_{A}\left(W_{1} \cap W_{2}\right)=\operatorname{comp}_{A} W_{1} \cap \operatorname{comp}_{A} W_{2}$;

(iii) $A\left(W_{1} \cap W_{2}\right) \leq A W_{1} \cap A W_{2}$;

(iv) $\operatorname{comp}_{A}\left(W_{1}+W_{2}\right) \geq \operatorname{comp}_{A} W_{1}+\operatorname{comp}_{A} W_{2}$, with equality if the sum $W_{1}+W_{2}$ is direct over $A$.

Proposition 2.4. Let $W \in\left[\begin{array}{l}V \\ { }_{*}\end{array}\right]_{k}$ and $W_{1} \in\left[\begin{array}{l}W \\ *\end{array}\right]_{A}$. Then there exists a subspace $U \in\left[\begin{array}{l}W \\ *\end{array}\right]_{k}$ such that $W=W_{1} \oplus_{A} U$.

Proof. Since $W_{1}$ is an $A$-submodule of the semisimple module $A W$, there exists an $A$-submodule $U_{1}$ of $A W$ such that $A W=W_{1} \oplus U_{1}$. Put $U=U_{1} \cap W$. From the fact that $W_{1} \leq W$, one easily sees that $W=W_{1} \oplus U, A U=U_{1}$, and $W_{1} \cap A U=W_{1} \cap U_{1}=0$, i.e., $W=W_{1} \oplus_{A} U$.

Remark. In general, an arbitrary complement $U$ of $W_{1}$ in $W$ over $k$ will not form a direct sum over $A$ with $W_{1}$. Examples are already easy to find in the case $\operatorname{dim}_{k} A=2$. However, we mention that if $W_{1}$ contains $\operatorname{comp}_{A} W$ and $\operatorname{dim}_{k} A=2$, then any complement of $W_{1}$ over $k$ will be a complement over $A$. This will be shown in Lemma 4.1 for the case $A=k \times k$. (See [4, Theorem 4.1(c)] for the case $A=k_{1}$, quadratic extension of $k$.) This fact does not hold when $\operatorname{dim}_{k} A \geq 3$.

Proposition 2.5. Let $W \in\left[\begin{array}{l}V \\ *\end{array}\right]_{k}$. Then, viewing each $e_{i} V$ as a vector space over $k_{i}$, we have

$$
\operatorname{comp}_{A} W=\bigoplus_{i=1}^{m} \operatorname{comp}_{k_{i}}\left(W \cap e_{i} V\right) \text { and } A W=\bigoplus_{i=1}^{m} k_{i} e_{i} W .
$$

In particular, if $A=k \times \cdots \times k$ ( $m$ factors), then

$$
\operatorname{comp}_{A} W=\bigoplus_{i=1}^{m}\left(W \cap e_{i} V\right) \text { and } A W=\bigoplus_{i=1}^{m} e_{i} W .
$$

Proposition 2.6. Let $W \in\left[\begin{array}{l}V \\ *\end{array}\right]_{k}$ and let $\left(W_{r}\right)_{r}$ be a family in $\left[\begin{array}{l}V \\ *\end{array}\right]_{k}$ such that $W=\bigoplus_{r} W_{r}$. This sum is direct over $A$ if and only if for each $i$ we have a direct sum $e_{i} W=\bigoplus_{r} e_{i} W_{r}$ over $k_{i}$. In particular, if $A=k \times \cdots \times k$ ( $m$ factors), then the sum $W=\bigoplus_{r} W_{r}$ is direct over $A$ if and only if $e_{i} W=\bigoplus_{r} e_{i} W_{r}$ for each $i$.

\section{THE MAIN PROBLEM}

We now assume $A=k_{1} \times \cdots \times k_{m}$ is finite dimensional over $k$, that is, each $k_{i} / k$ is a finite extension, and let $V$ be a finitely generated $A$-module. Viewing $V$ as a vector space over $k$ by restriction of scalars, we have the set $\left[\begin{array}{l}V \\ *\end{array}\right]_{k}$ of $k$-subspaces of $V$, partially ordered by inclusion. In general, given a poset $\mathscr{P}$ 
of finite height and a group $G$ acting on $\mathscr{P}$ by poset automorphisms, we can define the quotient poset $\mathscr{P} / G$ to be the set of orbits for the action of $G$ on $\mathscr{P}$, with the order relation defined by $\mathscr{O}_{1} \leq \mathscr{O}_{2}$ if there exists $x \in \mathscr{O}_{1}, y \in \mathscr{O}_{2}$ such that $x \leq y$ in $\mathscr{P}$. In our situation, the general linear group $\mathrm{GL}_{A}(V)$ acts by automorphisms on the poset $\left[\begin{array}{l}V \\ *\end{array}\right]_{k}$ and we want to investigate the quotient poset $\left[\begin{array}{l}V \\ *\end{array}\right]_{k} / \mathrm{GL}_{A}(V)$.

Since the poset $\left[\begin{array}{l}V \\ { }^{V}\end{array}\right]_{k}$ is ranked, so is the quotient poset. The cardinality of the $d$ th level of the quotient poset is the size of the double coset space

$$
P \backslash \mathrm{GL}_{k}(V) / \mathrm{GL}_{A}(V),
$$

where $P$ is a suitable maximal parabolic subgroup of $\mathrm{GL}_{k}(V)$. In terms of matrices, if $\left[k_{i}: k\right]=r_{i}, \operatorname{dim}_{A}(V)=\left(n_{1}, \ldots, n_{m}\right)$, and $\operatorname{dim}_{k} V=n=\sum_{i} r_{i} n_{i}$, we have to consider the double coset space

$$
P \backslash \mathrm{GL}(n, k) /\left(\mathrm{GL}\left(n_{1}, k_{1}\right) \times \cdots \times \mathrm{GL}\left(n_{m}, k_{m}\right)\right)
$$

with

$$
P=\left\{\left(\begin{array}{cc}
* & * \\
0_{d \times(n-d)} & *
\end{array}\right)\right\} \leq \mathrm{GL}(n, k),
$$

where we have chosen a suitable embedding of $\mathrm{GL}\left(n_{1}, k_{1}\right) \times \cdots \times \mathrm{GL}\left(n_{m}, k_{m}\right)$ into $\mathrm{GL}(n, k)$.

Our main result is the following:

Theorem 3.1. Let $A$ be a finite-dimensional commutative semisimple algebra over an infinite field $k$ and let $V$ be a faithful finitely generated $A$-module. Then the quotient poset $\left[\begin{array}{l}V \\ *\end{array}\right]_{k} / \mathrm{GL}_{A}(V)$ is finite if and only if $\operatorname{dim}_{k} A \leq 3$.

Proof. The case of $A$ an extension field of $k$ is considered in [4]. In $\S \S 4$ to 6, we show the finiteness of the quotient poset when $\operatorname{dim}_{k} A \leq 3$. Assume now that $\operatorname{dim}_{k} A \geq 4$ with $A=k_{1} \times \cdots \times k_{m}, m \geq 2$. To prove that the quotient poset is infinite, we may restrict our attention to the case $V$ free of rank 1 . We will show that the action of $\mathrm{GL}_{A}(A) \cong A^{\times}$on $\left[\begin{array}{l}A \\ 2\end{array}\right]_{k}$ has infinitely many orbits. The cases to consider are:

(i) $m \geq 4$;

(ii) $m=3$ with at least one simple component, say $k_{1}$, satisfying $\left[k_{1}: k\right] \geq$ 2 ;

(iii) $m=2$ with, say, $\left[k_{1}: k\right] \geq 3$;

(iv) $m=2$ with $\left[k_{1}: k\right]=\left[k_{2}: k\right]=2$.

In case (i), the subspaces $\left\langle e_{1}+e_{2}+e_{3}, e_{4}+e_{2}+a e_{3}\right\rangle_{k}\left(a \in k^{\times}\right)$are in different orbits as $a$ varies over $k^{\times}$. In case (ii), if 1 and $\alpha$ are $k$-independent elements in $k_{1}$, then $\left\langle e_{1}+e_{2}+e_{3}, \alpha e_{1}+a e_{2}\right\rangle_{k}\left(a \in k^{\times}\right)$are in different orbits. In case (iii), consider $\left\langle a e_{1}+\alpha e_{1}+e_{2}, \beta e_{1}\right\rangle_{k}\left(a \in k^{\times}\right)$for any fixed $k$-independent set $\{1, \alpha, \beta\}$ in $k_{1}$. We will supply details only in the remaining case (iv). 
For this last case, suppose $k_{1}=k(\alpha)$ and $k_{2}=k(\beta)$ with $\alpha^{2}=f_{1} \alpha+f_{2}$ and $\beta^{2}=g_{1} \beta+g_{2}\left(f_{1}, f_{2}, g_{1}, g_{2} \in k\right)$. We will show that for any distinct $a, b \in k$ satisfying $a+b+g_{1}-f_{1} \neq 0$, the subspaces $\left\langle e_{1}+e_{2}, \alpha e_{1}+(\beta+a) e_{2}\right\rangle_{k}$ and $\left\langle e_{1}+e_{2}, \alpha e_{1}+(\beta+b) e_{2}\right\rangle_{k}$ are in different orbits. Suppose not. Then we can find $\lambda_{1} \in k_{1}^{\times}, \lambda_{2} \in k_{2}^{\times}, p, q$ not both zero in $k$, and $r, s$ not both zero in $k$ such that

$$
\left\{\begin{array}{l}
\lambda_{1} e_{1}+\lambda_{2} e_{2}=p\left(e_{1}+e_{2}\right)+q\left(\alpha e_{1}+(\beta+b) e_{2}\right) \\
\alpha \lambda_{1} e_{1}+(\beta+a) \lambda_{2} e_{2}=r\left(e_{1}+e_{2}\right)+s\left(\alpha e_{1}+(\beta+b) e_{2}\right) .
\end{array}\right.
$$

By comparing the coefficients of $e_{1}$ and $e_{2}$ in (1), we obtain

$$
\left\{\begin{array}{l}
\lambda_{1}=p+q \alpha, \\
\lambda_{2}=p+q(\beta+b), \\
\alpha \lambda_{1}=r+s \alpha, \\
(\beta+a) \lambda_{2}=r+s(\beta+b) .
\end{array}\right.
$$

Eliminating $\lambda_{1}$ and $\lambda_{2}$ in (2), using $\alpha^{2}=f_{1} \alpha+f_{2}, \beta^{2}=g_{1} \beta+g_{2}$, and comparing the coefficients of $1, \alpha, \beta$, one derives

$$
\begin{gathered}
q\left(a+b+g_{1}-f_{1}\right)=0, \\
p(a-b)=q\left(f_{2}+f_{1} b-g_{2}-a b\right) .
\end{gathered}
$$

Hence $q=0$ and, since $p \neq 0$, we get the contradiction $a=b$.

Remark. The remaining sections of this paper and [4] actually show a much stronger result. Except in one exceptional case $(A=k \times k \times k$ and $k$ is the field with two elements), if $\operatorname{dim}_{k} A \leq 3$, the quotient poset $\left[\begin{array}{l}V \\ *\end{array}\right]_{k} / \mathrm{GL}_{A}(V)$ itself is independent of the fields $k, k_{1}, \ldots, k_{m}$ and only depends on $m$, the degrees $\left[k_{i}: k\right]$, and the dimension vector $\operatorname{dim}_{A} V$.

In the rest of this section, we state a few more general facts about the quotient poset $\left[\begin{array}{l}V \\ *\end{array}\right]_{k} / \mathrm{GL}_{A}(V)$, beginning with a duality result. $A$ and $V$ have the same meaning as above, with $k$ an arbitrary field; we do not assume $\operatorname{dim}_{k} A \leq 3$.

Theorem 3.2. The quotient poset $\left[\begin{array}{l}V \\ *\end{array}\right]_{k} / \mathrm{GL}_{A}(V)$ admits an involutory antiautomorphism. In particular, if $\left[\begin{array}{l}V \\ *\end{array}\right]_{k} / \mathrm{GL}_{A}(V)$ is finite, then it is rank-symmetric.

Proof. Choose an $A$-bilinear symmetric nonsingular pairing $B: V \times V \rightarrow A$. For each $i$, choose a nonzero $k$-linear map $\varphi_{i}: k_{i} \rightarrow k$, and let $\varphi: A \rightarrow k$ be defined by $\varphi\left(\sum \alpha_{i} e_{i}\right)=\sum \varphi_{i}\left(\alpha_{i}\right)\left(\alpha_{i} \in k_{i}\right)$. The map $B^{\prime}=\varphi \circ B$ is a $k$-bilinear symmetric nondegenerate pairing $V \times V \rightarrow k$. For $W \in\left[\begin{array}{l}V \\ *\end{array}\right]_{k}$, define $W^{\perp}=$ $\left\{v \in V \mid B^{\prime}(u, v)=0\right.$ for all $\left.u \in W\right\}$. Since $B^{\prime}$ is nondegenerate, $W^{\perp \perp}=W$ and the correspondence $W \mapsto W^{\perp}$ is an involutory antiautomorphism of $\left[\begin{array}{l}V \\ *\end{array}\right]_{k}$. Denote the transpose of an endomorphism $T \in \operatorname{End}_{A}(V)$ with respect to $B$ by ${ }^{t} T$, i.e., $B(T u, v)=B\left(u,{ }^{t} T v\right)(u, v \in V)$. Then it is easy to see that $(T W)^{\perp}={ }^{t}\left(T^{-1}\right)\left(W^{\perp}\right)$ for $W \in\left[\begin{array}{l}V \\ *\end{array}\right]_{k}$ and $T \in \mathrm{GL}_{A}(V)$. Therefore the 
correspondence $W \mapsto W^{\perp}$ induces an involutory antiautomorphism of the quotient poset $\left[\begin{array}{l}V \\ *\end{array}\right]_{k} / \mathrm{GL}_{A}(V)$.

The fact that the quotient poset is rank-symmetric if it is finite is also contained in the following result. Recall that a finite ranked poset is called Peck if it is rank-symmetric, rank-unimodal, and strongly Sperner (see [5]).

Theorem 3.3. If the poset $\left[\begin{array}{l}V \\ *\end{array}\right]_{k} / \mathrm{GL}_{A}(V)$ is finite, then it is Peck.

Proof. If $k$ is a finite field, then $\left[\begin{array}{l}V \\ *\end{array}\right]_{k}$ is unitary Peck [5, Theorem 2]. $\left[\begin{array}{l}V \\ *\end{array}\right]_{k} / \mathrm{GL}_{A}(V)$ is then Peck as a quotient of a unitary Peck poset [5, Theorem 1]. If $k$ is infinite but $\left[{ }^{V}\right]_{k} / \mathrm{GL}_{A}(V)$ is finite, Theorem 3.1 and the remark following its proof shows that $\left[\begin{array}{l}V \\ *\end{array}\right]_{k} / \mathrm{GL}_{A}(V)$ is isomorphic to a quotient poset of the same form with $k$ finite. So the result is also true in this case.

\section{THE CASE $A=k \times k$}

In this section $A$ is the algebra $k \times k$ and $V$ is an $A$-module of finite bidimension $\left(n_{1}, n_{2}\right)$. We begin with the result announced in the remark following Proposition 2.4 .

Lemma 4.1. Let $W \in\left[{ }^{V}\right]_{k}$. If $W=U \oplus Y$ for some $k$-subspaces $U$ and $Y$ with $\operatorname{comp}_{A} W \leq U$, then $W=U \oplus_{A} Y$. In particular, any $k$-subspace complementary to comp ${ }_{A} W$ in $W$ forms a direct sum over $A$ with $\operatorname{comp}_{A} W$, and if $\operatorname{comp}_{A} W=0$, any direct sum $W=U \oplus Y$ is direct over $A$.

Proof. We have to show that $e_{i} U \cap e_{i} Y=0$ for $i=1,2$. Say $i=1$. If $v \in$ $e_{1} U \cap e_{1} Y$, then $v=e_{1} u=e_{1} y$ for some $u \in U, y \in Y$. Hence $e_{1}(u-y)=0$ and $u-y \in e_{2} V \cap W \leq \operatorname{comp}_{A} W \leq U$, which implies $y \in U \cap Y=0$ and $v=0$.

Define the type of a $k$-subspace $W$ of $V$ to be the triple of nonnegative integers

$$
\operatorname{type}(W)=\left(\operatorname{dim}_{k}\left(W \cap e_{1} W\right), \operatorname{dim}_{k}\left(W \cap e_{2} W\right), \operatorname{dim}_{k}\left(W / \operatorname{comp}_{A} W\right)\right) .
$$

The type of a subspace is clearly invariant under the action of $\mathrm{GL}_{A}(V)$ on $\left[\begin{array}{l}V \\ *\end{array}\right]_{k}$. We will shortly see that the parameters of the type constitute a complete set of invariants for this action.

Theorem 4.2. Let $W \in\left[\begin{array}{l}V \\ *\end{array}\right]_{k}$ with type $(W)=\left(m_{1}, m_{2}, t\right)$. There exist $k$ independent vectors $v_{1}^{(i)}, \ldots, v_{m_{i}}^{(i)} ; z_{1}^{(i)}, \ldots, z_{t}^{(i)}$ in $e_{i} V(i=1,2)$ such that

(1) $W=\left\langle v_{1}^{(1)}, \ldots, v_{m_{1}}^{(1)}\right\rangle_{k} \oplus_{A}\left\langle v_{1}^{(2)}, \ldots, v_{m_{2}}^{(2)}\right\rangle_{k} \oplus_{A}\left\langle z_{1}^{(1)}+z_{1}^{(2)}, \ldots, z_{t}^{(1)}+z_{t}^{(2)}\right\rangle_{k}$.

The vectors $v_{1}^{(i)}, \ldots, v_{m_{i}}^{(i)}$ form a basis of $e_{i} W \cap W$ over $k(i=1,2)$.

Proof. Let $W \in\left[\begin{array}{l}V \\ *\end{array}\right]_{k}$ wih type $(W)=\left(m_{1}, m_{2}, t\right)$. By Proposition 2.4, we can write $W=\operatorname{comp}_{A} W \oplus_{A} U$ for some subspace $U \in\left[\begin{array}{c}W \\ t\end{array}\right]_{k}$ (or use Lemma 
4.1). We have $\operatorname{comp}_{A} W=\left(e_{1} W \cap W\right) \oplus\left(e_{2} W \cap W\right)$. Let $\left\{v_{1}^{(i)}, \ldots, v_{m_{i}}^{(i)}\right\}$ be a basis of $e_{i} W \cap W$ over $k(i=1,2)$. Let $\left\{z_{1}, \ldots, z_{t}\right\}$ be a basis of $U$ over $k$; put $z_{p}^{(i)}=e_{i} z_{p}(p=1, \ldots, t ; i=1,2)$ so that $z_{p}=z_{p}^{(1)}+z_{p}^{(2)}$.

The vectors $z_{1}^{(i)}, \ldots, z_{t}^{(i)}(i=1,2)$ are linearly independent over $k$. For example, for $i=1$, suppose $a_{1}, \ldots, a_{t} \in k$ and $\sum_{p} a_{p} z_{p}^{(1)}=0$. Then $\sum_{p} a_{p} z_{p}=\sum_{p} a_{p} z_{p}^{(2)} \in e_{2} V$. Since $U \cap e_{2} V \leq U \cap \operatorname{comp}_{A} W=0$, we have $\sum_{p} a_{p} z_{p}=0$, which implies $a_{p}=0$ for all $p$. Since comp ${ }_{A} W \cap A U=0$, the vectors $v_{1}^{(i)}, \ldots, v_{m_{i}}^{(i)} ; z_{1}^{(i)}, \ldots, z_{t}^{(i)}$ are all independent over $k(i=1,2)$.

The above theorem shows that if $W \in\left[\begin{array}{l}V \\ *\end{array}\right]_{k}$ has type $\left(m_{1}, m_{2}, t\right)$ then

$$
\operatorname{dim}_{A} \operatorname{comp}_{A} W=\left(m_{1}, m_{2}\right), \quad \operatorname{dim}_{A} A W=\left(m_{1}+t, m_{2}+t\right)
$$

and

$$
\begin{gathered}
\operatorname{dim}_{k} \operatorname{comp}_{A} W=m_{1}+m_{2} \\
\operatorname{dim}_{k} W=m_{1}+m_{2}+t, \quad \operatorname{dim}_{k} A W=m_{1}+m_{2}+2 t
\end{gathered}
$$

In particular,

$$
m_{i}+t \leq n_{i} \quad(i=1,2)
$$

Conversely, given any nonnegative integers $m_{1}, m_{2}, t$ satisfying (2), there exists a subspace $W \in\left[\begin{array}{l}V \\ *\end{array}\right]_{k}$ of type $\left(m_{1}, m_{2}, t\right)$, namely the subspace $W$ given by (1) in Theorem 4.2 for any particular choice of $k$-independent vectors $v_{1}^{(i)}, \ldots, v_{m_{i}}^{(i)} ; z_{1}^{(i)}, \ldots, z_{t}^{(i)}$ in $e_{i} V(i=1,2)$.

Theorem 4.3. Two $k$-subspaces of $V$ are in the same orbit for $\mathrm{GL}_{A}(V)$ if and only if they have the same type.

Proof. We have already noticed that the type of a subspace is invariant under the action of $\mathrm{GL}_{A}(V)$. Conversely, if $W$ and $W^{\prime}$ are two $k$-subspaces of $V$ of the same type, both subspaces can be written in the same standard form given in Theorem 4.2 with respect to suitable bases $B_{1}$ and $B_{1}^{\prime}$ of $e_{1} V$ and $B_{2}$ and $B_{2}^{\prime}$ of $e_{2} V$ over $k$. The $A$-automorphism of $V$ that sends the bases $B_{1}$ and $B_{2}$ to $B_{1}^{\prime}$ and $B_{2}^{\prime}$ respectively will map $W$ onto $W^{\prime}$.

We now have a one-to-one correspondence between the set $\left\{\left(m_{1}, m_{2}, t\right) \in\right.$ $\left.\mathbb{N}^{3} \mid m_{i}+t \leq n_{i}, i=1,2\right\}$ and the set of orbits for the action of $\mathrm{GL}_{A}(V)$ on $\left[\begin{array}{l}V \\ { }_{*}\end{array}\right]_{k}$. It is readily established that, for triples $\left(m_{1}, m_{2}, t\right)$ and $\left(m_{1}^{\prime}, m_{2}^{\prime}, t^{\prime}\right)$ $\in \mathbb{N}^{3}$ satisfying (2), there exist subspaces $W, W^{\prime} \in\left[\begin{array}{l}V \\ *\end{array}\right]_{k}$ with $\operatorname{type}(W)=$ $\left(m_{1}, m_{2}, t\right), \operatorname{type}\left(W^{\prime}\right)=\left(m_{1}^{\prime}, m_{2}^{\prime}, t^{\prime}\right)$, and $W \leq W^{\prime}$ if and only if the following four inequalities hold:

$$
\begin{gathered}
m_{i} \leq m_{i}^{\prime} \quad(i=1,2), \\
m_{i}+t \leq m_{i}^{\prime}+t^{\prime} \quad(i=1,2) .
\end{gathered}
$$


We are now in a position to state the main theorem of this section.

Theorem 4.4. Let $A$ be the algebra $k \times k$ over a field $k$ and let $V$ be an A-module of finite bidimension $\left(n_{1}, n_{2}\right)$. The quotient poset $\left[\begin{array}{l}V \\ *\end{array}\right]_{k} / \mathrm{GL}_{A}(V)$ is finite and independent of the field $k$. It is isomorphic to the poset

$$
\mathscr{P}_{n_{1}, n_{2}}=\left\{\left(m_{1}, m_{2}, t\right) \in \mathbb{N}^{3} \mid m_{i}+t \leq n_{i}, i=1,2\right\}
$$

with the order relation between triples given by (3).

Next, we state additional information that we have gathered about the poset $\mathscr{P}_{n_{1}, n_{2}}$. The situation being symmetric in $n_{1}$ and $n_{2}$, we shall now assume $n_{1} \leq n_{2}$. From $\S 3$, we already know that $\mathscr{P}_{n_{1}, n_{2}}$ is ranked and Peck. It has height $n_{1}+n_{2}$, with minimum element $(0,0,0)$, maximum element $\left(n_{1}, n_{2}, 0\right)$, and the rank of a triple $\left(m_{1}, m_{2}, t\right)$ equal to $m_{1}+m_{2}+t$. It is not a lattice in general; for instance, the two triples $(1,2,0)$ and $(1,1,1)$ of rank 3 both cover the two triples $(1,1,0)$ and $(0,1,1)$ of rank 2 . If $\mathscr{P}_{n_{1}, n_{2}}^{(d)}$ denotes the $d$ th level of the poset, then

$$
\left|\mathscr{P}_{n_{1}, n_{2}}^{(d)}\right|= \begin{cases}\left(\begin{array}{c}
d+2 \\
2
\end{array}\right) & \text { if } d \leq n_{1} \leq n_{2} \\
\left(\begin{array}{c}
n_{1}+2 \\
2
\end{array}\right) & \text { if } n_{1} \leq d \leq n_{2} \\
\left(\begin{array}{c}
n_{1}+n_{2}-d+2 \\
2
\end{array}\right) & \text { if } n_{1} \leq n_{2} \leq d\end{cases}
$$

The cardinality of the poset is given by

$$
\left|\mathscr{P}_{n_{1}, n_{2}}\right|=\frac{1}{6}\left(n_{1}+1\right)\left(n_{1}+2\right)\left(3 n_{2}-n_{1}+3\right) .
$$

We have the following rational generating function:

$$
\sum_{n_{1}, n_{2}, d \geq 0} \mathscr{P}_{n_{1}, n_{2}}^{(d)} x^{n_{1}} y^{n_{2}} q^{d}=\frac{1}{(1-x)(1-y)(1-x q)(1-y q)(1-x y q)} .
$$

Putting $q=1$ gives the special case

$$
\sum_{n_{1}, n_{2} \geq 0}\left|\mathscr{P}_{n_{1}, n_{2}}\right| x^{n_{1}} y^{n_{2}}=\frac{1}{(1-x)^{2}(1-y)^{2}(1-x y)}
$$

\section{THE CASE $A=k \times k \times k$}

In this section $A$ is the algebra $k \times k \times k$ and $V$ is an $A$-module of finite tridimension $\left(n_{1}, n_{2}, n_{3}\right)$. Throughout this section $\{i, j, l\}$ will always indicate a permutation of $\{1,2,3\}$.

For a $k$-subspace $W$ of $V$ we define

$$
\begin{aligned}
\Lambda_{i}(W) & =W \cap e_{i} W=W \cap e_{i} V, \\
\Lambda_{i j}(W) & =W \cap\left(e_{i}+e_{j}\right) W=W \cap\left(e_{i}+e_{j}\right) V, \\
\tilde{\Lambda}_{i j}(W) & =\Lambda_{i j}(W) \cap\left(\Lambda_{i l}(W)+\Lambda_{j l}(W)\right), \\
\widetilde{\Delta}(W) & =\widetilde{\Lambda}_{12}(W)+\tilde{\Lambda}_{13}(W)+\tilde{\Lambda}_{23}(W), \\
\Delta(W) & =\Lambda_{12}(W)+\Lambda_{13}(W)+\Lambda_{23}(W),
\end{aligned}
$$


and let the type of $W$ be the 8-tuple of nonnegative integers

$$
\begin{aligned}
\operatorname{type}(W)=\left(\operatorname{dim}_{k} \Lambda_{i}(W)(i=1,2,3) ; \frac{1}{2} \operatorname{dim}_{k}\left(\tilde{\Delta}(W) / \operatorname{comp}_{A} W\right) ;\right. \\
\\
\left.\operatorname{dim}_{k}\left(\Lambda_{j l}(W) / \tilde{\Lambda}_{j l}(W)\right)(i=1,2,3) ; \operatorname{dim}_{k}(W / \Delta(W))\right) .
\end{aligned}
$$

We will see in Lemma 5.3 that $\operatorname{dim}_{k}\left(\widetilde{\Delta}(W) / \operatorname{comp}_{A} W\right)$ is always an even integer, so that type $(W)$ is well defined. The parameters in type $(W)$ are invariant under the action of $\mathrm{GL}_{A}(V)$ on $\left[\begin{array}{l}V \\ *\end{array}\right]_{k}$ and will form a complete set of invariants for this action.

It will sometimes be convenient to use the following alternative notation for the type of a subspace. We introduce independent indeterminates $M_{1}, M_{2}, M_{3}$, $R, S_{1}, S_{2}, S_{3}, T$ and encode the type $\left(m_{1}, m_{2}, m_{3} ; r ; s_{1}, s_{2}, s_{3} ; t\right)$ of a subspace by the monomial $M_{1}^{m_{1}} M_{2}^{m_{2}} M_{3}^{m_{3}} R^{r} S_{1}^{s_{1}} S_{2}^{s_{2}} S_{3}^{s_{3}} T^{t}$, omitting the exponents equal to 1 and the variables whose exponent is 0 .

Theorem 5.1. Let $W \in\left[\begin{array}{l}V \\ *\end{array}\right]_{k}$ with type $(W)=\left(m_{1}, m_{2}, m_{3} ; r ; s_{1}, s_{2}, s_{3} ; t\right)$. There exist $k$-independent vectors $v_{1}^{(i)}, \ldots, v_{m_{i}}^{(i)} ; x_{1}^{(i)}, \ldots, x_{r}^{(i)} ; y_{1}^{(i, j)}, \ldots$, $y_{s_{j}}^{(i, j)} ; y_{1}^{(i, l)}, \ldots, y_{s_{l}}^{(i, l)} ; z_{1}^{(i)}, \ldots, z_{t}^{(i)}$ in $e_{i} V(i=1,2,3)$ such that $W$ admits the direct sum decomposition over $A$

$$
W=\left(\bigoplus_{i=1}^{3} \Lambda_{i}(W)\right) \oplus_{A} X \oplus_{A}\left(\bigoplus_{i=1}^{3} Y_{i}\right) \oplus_{A} Z
$$

(where both sums $\bigoplus_{i=1}^{3}$ are also direct over $A$ ) with

$$
\begin{aligned}
\Lambda_{i}(W) & =\left\langle v_{1}^{(i)}, \ldots, v_{m_{i}}^{(i)}\right\rangle_{k}, \\
X & =\bigoplus_{p=1}^{r}\left\langle x_{p}^{(1)}-x_{p}^{(2)}, x_{p}^{(1)}-x_{p}^{(3)}\right\rangle_{k}, \\
Y_{i} & =\bigoplus_{p=1}^{s_{i}}\left\langle y_{p}^{(j, i)}+y_{p}^{(l, i)}\right\rangle_{k}, \\
Z & =\bigoplus_{p=1}^{t}\left\langle z_{p}^{(1)}+z_{p}^{(2)}+z_{p}^{(3)}\right\rangle_{k} .
\end{aligned}
$$

Moreover, we have

$$
\begin{aligned}
\operatorname{comp}_{A} W & =\Lambda_{1}(W) \oplus_{A} \Lambda_{2}(W) \oplus_{A} \Lambda_{3}(W), \\
\tilde{\Lambda}_{i j}(W) & =\Lambda_{i}(W) \oplus_{A} \Lambda_{j}(W) \oplus_{A}\left\langle x_{1}^{(i)}-x_{1}^{(j)}, \ldots, x_{r}^{(i)}-x_{r}^{(j)}\right\rangle_{k}, \\
\Lambda_{i j}(W) & =\widetilde{\Lambda}_{i j}(W) \oplus_{A} Y_{l}, \\
\widetilde{\Delta}(W) & =\operatorname{comp}_{A} W \oplus_{A} X, \\
\Delta(W) & =\widetilde{\Delta}(W) \oplus_{A} Y_{1} \oplus_{A} Y_{2} \oplus_{A} Y_{3} .
\end{aligned}
$$


Remarks. (1) The subspace $X$ is characterized by a "trace condition": $\left\langle x^{(1)}-x^{(2)}, x^{(1)}-x^{(3)}\right\rangle_{k}=\left\{a x^{(1)}+b x^{(2)}+c x^{(3)} \mid a, b, c \in k ; a+b+c=0\right\}$.

(2) The fourth parameter of type $(W)$ is given by the equivalent expressions

$$
\begin{aligned}
r & =\frac{1}{2} \operatorname{dim}_{k} X=\frac{1}{2} \operatorname{dim}_{k}\left(\tilde{\Delta}(W) / \operatorname{comp}_{A} W\right) \\
& =\operatorname{dim}_{k}\left(\tilde{\Lambda}_{i j}(W) /\left(\Lambda_{i}(W) \oplus \Lambda_{j}(W)\right)\right) .
\end{aligned}
$$

Proof of Theorem 5.1. The proof will involve a series of lemmas. To begin with, $\operatorname{comp}_{A} W=\bigoplus_{i=1}^{3} \Lambda_{i}(W)$ by Proposition 2.5. For each $i=1,2,3$, choose a basis $\left\{v_{1}^{(i)}, \ldots, v_{m_{i}}^{(i)}\right\}$ of $\Lambda_{i}(W)$ over $k$. By Proposition 2.4, we may find a subspace $U \in\left[\begin{array}{c}W \\ *\end{array}\right]_{k}$ such that $W=\operatorname{comp}_{A} W \oplus_{A} U$. Necessarily, $\operatorname{comp}_{A} U=0$. So we are allowed to make the simplifying assumption that $\operatorname{comp}_{A} W=0$, which will hold until the end of the proof.

For short, let us write $W_{i j}$ for $\Lambda_{i j}(W)$ and $\widetilde{W}_{i j}$ for $\tilde{\Lambda}_{i j}(W)$. We first examine the subspace $X=\widetilde{\Delta}(W)$.

Lemma 5.2. (i) $W_{i j} \cap W_{i l}=0$.

(ii) The projection $v \mapsto e_{i} v$ from $W_{i j}$ onto $e_{i} W_{i j}$ is a $k$-linear isomorphism. Proof. (i) $W_{i j} \cap W_{i l} \leq W \cap e_{i} V=\Lambda_{i}(W) \leq \operatorname{comp}_{A} W=0$.

(ii) The kernel of the projection is $W_{i j} \cap\left(e_{j}+e_{l}\right) V=W_{i j} \cap W_{j l}=0$ by part (i).

Lemma 5.3. (i) $\operatorname{dim}_{k} \widetilde{W}_{12}=\operatorname{dim}_{k} \widetilde{W}_{13}=\operatorname{dim}_{k} \widetilde{W}_{23}=r$.

(ii) There exist $k$-independent vectors $x_{1}^{(i)}, \ldots, x_{r}^{(i)}$ in $e_{i} V(i=1,2,3)$ such that

$$
\widetilde{W}_{i j}=\left\langle x_{1}^{(i)}-x_{1}^{(j)}, \ldots, x_{r}^{(i)}-x_{r}^{(j)}\right\rangle_{k}
$$

(iii) $\widetilde{\Delta}(W)=\widetilde{W}_{i j} \oplus \widetilde{W}_{i l}$ and hence $\operatorname{dim}_{k} \widetilde{\Delta}(W)$ is even.

(iv) $W_{i j} \cap \widetilde{\Delta}(W)=\widetilde{W}_{i j}$.

Proof. (i) To prove that the three dimensions are equal, it suffices to show, by symmetry, that $\operatorname{dim}_{k} \widetilde{W}_{12} \leq \operatorname{dim}_{k} \widetilde{W}_{13}$. Let $n=\operatorname{dim}_{k} \widetilde{W}_{12}$ and pick a basis $\left\{v_{1}, \ldots, v_{n}\right\}$ of $\widetilde{W}_{12}$ over $k$. By definition of $\widetilde{\Lambda}_{12}(W)$ and Lemma 5.2(i), each $v_{p}(p=1, \ldots, n)$ is written uniquely in the form $v_{p}=y_{p}-z_{p}$ with $y_{p} \in W_{13}$ and $z_{p} \in W_{23}$. Then $y_{p}=v_{p}+z_{p} \in W_{13} \cap\left(W_{12}+W_{23}\right)=\widetilde{W}_{13}$. Claim: the $y_{p}$ 's are independent over $k$. Suppose $\sum_{p} m_{p} y_{p}=0$ for some scalars $m_{p} \in k$. Then $\sum_{p} m_{p} v_{p}=-\sum_{p} m_{p} z_{p} \in W_{12} \cap W_{23}=0$ by Lemma 5.2(i). Hence $m_{p}=0$ for all $p$. This proves that the three subspaces $\widetilde{W}_{12}, \widetilde{W}_{13}, \widetilde{W}_{23}$ have the same dimension $n$. The fact that $n=r$ is shown in (iii) below.

(ii) Keeping the same notation as above, $\left\{y_{1}, \ldots, y_{n}\right\}$ is a basis of $\widetilde{W}_{13}$ over $k$. Similarly, $\left\{z_{1}, \ldots, z_{n}\right\}$ is a basis of $\widetilde{W}_{23}$ over $k$. For each $p=1, \ldots, n$, put $x_{p}^{(1)}=e_{1} v_{p}, x_{p}^{(2)}=-e_{2} v_{p}, x_{p}^{(3)}=-e_{3} y_{p}$. By Lemma 5.2(ii), the vectors 
$x_{1}^{(i)}, \ldots, x_{n}^{(i)}$ in $e_{i} V$ are independent over $k(i=1,2,3)$. We have $v_{p}=$ $e_{1} v_{p}+e_{2} v_{p}=x_{p}^{(1)}-x_{p}^{(2)}$. Also $e_{1} y_{p}=e_{1} v_{p}+e_{1} z_{p}=x_{p}^{(1)}$ since $e_{1} z_{p}=0$; hence $y_{p}=e_{1} y_{p}+e_{3} y_{p}=x_{p}^{(1)}-x_{p}^{(3)}$. Finally, $e_{2} z_{p}=e_{2} y_{p}-e_{2} v_{p}=x_{p}^{(2)}$ and $e_{3} z_{p}=e_{3} y_{p}-e_{3} v_{p}=-x_{p}^{(3)}$, so that $z_{p}=x_{p}^{(2)}-x_{p}^{(3)}$. So each $\widetilde{W}_{i j}$ has the form stated above.

(iii) We now have $\widetilde{W}_{j l} \leq \widetilde{W}_{i j}+\widetilde{W}_{i l}$. Since this last sum is direct by Lemma 5.2(i), we have $\widetilde{\Delta}(W)=\widetilde{W}_{i j}+\widetilde{W}_{i l}+\widetilde{W}_{j l}=\widetilde{W}_{i j} \oplus \widetilde{W}_{i l}$. Also $\operatorname{dim}_{k} \tilde{\Delta}(W)=$ $\operatorname{dim}_{k} \widetilde{W}_{i j}+\operatorname{dim}_{k} \widetilde{W}_{i l}=2 n$, so that $r=\frac{1}{2} \operatorname{dim}_{k} \widetilde{\Delta}(W)=n$.

(iv) follows easily from (iii) and Lemma 5.2(i).

For each $i$, pick a complementary subspace $Y_{i}$ of $\widetilde{W}_{j l}$ in $W_{j l}$, i.e., $W_{j l}=$ $\widetilde{W}_{j l} \oplus Y_{i}$. Each $Y_{i}$ has dimension $s_{i}$ over $k$.

Lemma 5.4. We have the following direct sum decompositions over $A$ :

(i) $W_{j l}=\widetilde{W}_{j l} \oplus_{A} Y_{i}$;

(ii) $\Delta(W)=\widetilde{\Delta}(W) \oplus_{A} Y_{1} \oplus_{A} Y_{2} \oplus_{A} Y_{3}$.

Proof. (i) is a direct consequence of (ii), which we prove directly. Clearly, $\Delta(W)=\widetilde{\Delta}(W)+Y_{1}+Y_{2}+Y_{3}$. We first show that this sum is direct, i.e.,

$$
\Delta(W)=\tilde{\Delta}(W) \oplus Y_{1} \oplus Y_{2} \oplus Y_{3} .
$$

Let $u \in \tilde{\Delta}(W), v_{i} \in Y_{i}(i=1,2,3)$ such that $u+v_{1}+v_{2}+v_{3}=0$. Since $v_{1} \in W_{23}, v_{2} \in W_{13}$, and $\widetilde{\Delta}(W) \leq W_{13}+W_{23}$ by Lemma 5.3(iii), we have $v_{3}=-u-v_{1}-v_{2} \in W_{13}+W_{23}$. Hence $v_{3} \in Y_{3} \leq W_{12}$ implies $v_{3} \in W_{12} \cap$ $\left(W_{13}+W_{23}\right)=\widetilde{W}_{12}$ and this forces $v_{3}=0$ by the definition of $Y_{3}$. Similarly, $v_{1}=v_{2}=0$ and therefore $u=0$. We now show that the direct sum (1) is direct over $A$. By Proposition 2.6, it is equivalent to show that

$$
e_{i} \Delta(W)=e_{i} \tilde{\Delta}(W) \oplus e_{i} Y_{1} \oplus e_{i} Y_{2} \oplus e_{i} Y_{3}
$$

for $i=1,2,3$. Let us do the case $i=1$. Since $e_{1} Y_{1}=0$, the problem is to show that the sum $e_{1} \tilde{\Delta}(W)+e_{1} Y_{2}+e_{1} Y_{3}$ is direct. Let $u \in \widetilde{\Delta}(W), v_{2} \in$ $Y_{2}, v_{3} \in Y_{3}$ with $e_{1} u+e_{1} v_{2}+e_{1} v_{3}=0$. Since $e_{1}\left(u+v_{2}+v_{3}\right)=0$, we have $u+v_{2}+v_{3} \in W \cap\left(e_{2}+e_{3}\right) V=W_{23}=\widetilde{W}_{23} \oplus Y_{1}$. This implies $v_{2}+v_{3} \epsilon$ $\widetilde{\Delta}(W)+W_{23}=\widetilde{\Delta}(W) \oplus Y_{1}$ and hence $v_{2}=v_{3}=0$ because of the direct sum (1). Finally, $e_{1} v_{2}=e_{1} v_{3}=0$ implies $e_{1} u=0$, which completes the proof.

For each $i=1,2,3$, choose a basis $\left\{y_{1}^{(i)}, \ldots, y_{s_{s}}^{(i)}\right\}$ of $Y_{i}$ over $k$. Put $y_{p}^{(j, i)}=e_{j} y_{p}^{(i)}, y_{p}^{(l, i)}=e_{l} y_{p}^{(i)}$, so that $y_{p}^{(i)}=y_{p}^{(j, i)}+y_{p}^{(l, i)}\left(p=1, \ldots, s_{i}\right)$. By Lemma 5.2(ii), the vectors $y_{p}^{(j, i)}\left(p=1, \ldots, s_{i}\right)$ are $k$-independent in $e_{j} V$.

Finally, choose a complementary subspace $Z$ of $\Delta(W)$ in $W$, i.e., $W=$ $\Delta(W) \oplus Z$. The subspace $Z$ has dimension $t$ over $k$.

Lemma 5.5. (i) $W=\Delta(W) \oplus_{A} Z$.

(ii) For each $i=1,2,3$, the projection $v \mapsto e_{i} v$ from $Z$ onto $e_{i} Z$ is a $k$-linear isomorphism. 
Proof. (i) By Proposition 2.6, we have to show that $e_{i} W=e_{i} \Delta(W) \oplus e_{i} Z$. Since $e_{i} W=e_{i} \Delta(W)+e_{i} Z$, this amounts to showing $e_{i} \Delta(W) \cap e_{i} Z=0$. Suppose $u \in \Delta(W), v \in Z$, and $e_{i} u=e_{i} v$. Then $e_{i}(v-u)=0$ implies $v-u \in W \cap\left(e_{j}+e_{l}\right) V=W_{j l} \leq \Delta(W)$ and hence $v \in \Delta(W)$. So $v=0$ because of the direct sum $\Delta(W) \oplus Z$.

(ii) The kernel of this projection is $Z \cap\left(e_{j}+e_{l}\right) V \leq W \cap\left(e_{j}+e_{l}\right) V=W_{j l} \leq$ $\Delta(W)$. Hence this kernel is $Z \cap \Delta(W)=0$.

The proof of Theorem 5.1 is now completed by choosing a basis $\left\{z_{1}, \ldots, z_{t}\right\}$ of $Z$ over $k$ and putting $z_{p}^{(i)}=e_{i} z_{p}(i=1,2,3 ; p=1, \ldots, t)$. The vectors $z_{1}^{(i)}, \ldots, z_{t}^{(i)}$ in $e_{i} V$ are $k$-independent by Lemma 5.5(ii). Moreover, the vectors $x_{1}^{(i)}, \ldots, x_{r}^{(i)} ; y_{1}^{(i, j)}, \ldots, y_{s_{j}}^{(i, j)} ; y_{1}^{(i, l)}, \ldots, y_{s_{l}}^{(i, l)} ; z_{1}^{(i)}, \ldots, z_{t}^{(i)}$ in $e_{i} V$ are all independent over $k$ because the direct sums in Lemmas 5.4 and 5.5(i) are direct over $A$.

Remark. We record the following, which may be deduced from the preceding proof. For a $k$-subspace $W$ of $V$,

$$
\begin{aligned}
\Lambda_{i}(W) & =\Lambda_{i j}(W) \cap \Lambda_{i l}(W), \\
\widetilde{\Delta}(W) & =\tilde{\Lambda}_{i j}(W)+\tilde{\Lambda}_{i l}(W), \\
\tilde{\Lambda}_{i j}(W) & =\Lambda_{i j}(W) \cap \tilde{\Delta}(W) .
\end{aligned}
$$

Remark. If type $(W)=\left(m_{1}, m_{2}, m_{3} ; r ; s_{1}, s_{2}, s_{3} ; t\right)$ then

$$
\begin{aligned}
& \operatorname{dim}_{A} \operatorname{comp}_{A} W=\left(m_{1}, m_{2}, m_{3}\right), \\
& \operatorname{dim}_{A} A W=\left(m_{1}+r+s_{2}+s_{3}+t, m_{2}+r+s_{1}+s_{3}+t, m_{3}+r+s_{1}+s_{2}+t\right)
\end{aligned}
$$

and

$$
\begin{aligned}
& \operatorname{dim}_{k} \operatorname{comp}_{A} W=m_{1}+m_{2}+m_{3}, \\
& \operatorname{dim}_{k} \tilde{\Lambda}_{i j}(W)=m_{i}+m_{j}+r, \\
& \operatorname{dim}_{k} \Lambda_{i j}(W)=m_{i}+m_{j}+r+s_{l}, \\
& \operatorname{dim}_{k} \tilde{\Delta}(W)=m_{1}+m_{2}+m_{3}+2 r, \\
& \operatorname{dim}_{k} \Delta(W)=m_{1}+m_{2}+m_{3}+2 r+s_{1}+s_{2}+s_{3}, \\
& \operatorname{dim}_{k} W=m_{1}+m_{2}+m_{3}+2 r+s_{1}+s_{2}+s_{3}+t, \\
& \operatorname{dim}_{k} A W=m_{1}+m_{2}+m_{3}+3 r+2 s_{1}+2 s_{2}+2 s_{3}+3 t .
\end{aligned}
$$

From the expression for $\operatorname{dim}_{A} A W$ and Theorem 5.1, we see that an 8-tuple $\left(m_{1}, m_{2}, m_{3} ; r ; s_{1}, s_{2}, s_{3} ; t\right) \in \mathbb{N}^{8}$ is the type of some $k$-subspace of $V$ if and only if

$$
m_{i}+r+s_{j}+s_{l}+t \leq n_{i} \quad \text { for } i=1,2,3 .
$$

The following result is proved like Theorem 4.3.

Theorem 5.6. Two k-subspaces of $V$ are in the same orbit for $\mathrm{GL}_{A}(V)$ if and only if they have the same type. 
We may now state the main result of this section.

Theorem 5.7. Let $A$ be the algebra $k \times k \times k$, where $k$ is a field different from the field $\mathbf{F}_{2}$ with two elements, and let $V$ be an $A$-module of finite tridimension $\left(n_{1}, n_{2}, n_{3}\right)$. The quotient poset $\left[\begin{array}{l}V \\ *\end{array}\right]_{k} / \mathrm{GL}_{A}(V)$ is finite and independent of the field $k$. It is isomorphic to the poset

$$
\begin{array}{r}
\mathscr{P}_{n_{1} n_{2}, n_{3}}=\left\{\left(m_{1}, m_{2}, m_{3} ; r ; s_{1}, s_{2}, s_{3} ; t\right) \in \mathbb{N}^{8} \mid m_{i}+r+s_{j}+s_{l}+t \leq n_{i}\right. \\
\text { for } i=1,2,3\},
\end{array}
$$

with the order relation

$$
\left(m_{1}, m_{2}, m_{3} ; r ; s_{1}, s_{2}, s_{3} ; t\right) \leq\left(m_{1}^{\prime}, m_{2}^{\prime}, m_{3}^{\prime} ; r^{\prime} ; s_{1}^{\prime}, s_{2}^{\prime}, s_{3}^{\prime} ; t^{\prime}\right)
$$

given by the following 18 inequalities:

$$
\begin{aligned}
m_{i} & \leq m_{i}^{\prime} & & (i=1,2,3), \\
m_{i}+r & \leq m_{i}^{\prime}+r^{\prime} & & (i=1,2,3), \\
m_{i}+r+s_{j} & \leq m_{i}^{\prime}+r^{\prime}+s_{j}^{\prime} & & (\text { all distinct } i, j \in\{1,2,3\}), \\
m_{i}+r+s_{j}+s_{l} & \leq m_{i}^{\prime}+r^{\prime}+s_{j}^{\prime}+s_{l}^{\prime} & & (i=1,2,3), \\
m_{i}+r+s_{j}+s_{l}+t & \leq m_{i}^{\prime}+r^{\prime}+s_{j}^{\prime}+s_{l}^{\prime}+t^{\prime} & & (i=1,2,3) .
\end{aligned}
$$

Proof. By the preceding remarks and Theorem 5.6, we have a one-to-one correspondence between $\mathscr{P}_{n_{1}, n_{2}, n_{3}}$ and the set of orbits for the action of $\mathrm{GL}_{A}(V)$ on $\left[\begin{array}{l}V \\ *\end{array}\right]_{k}$. To prove the necessity of the above 18 inequalities for the order relation in $\mathscr{P}_{n_{1}, n_{2}, n_{3}}$, observe that the operators $\Lambda_{i}, \Lambda_{i j}, \widetilde{\Lambda}_{i j}, \widetilde{\Delta}$, and $\Delta$ preserve inclusion of subspaces and that the left-hand members of the 18 inequalities are equal to $\operatorname{dim}_{k} \Lambda_{i}(W), \operatorname{dim}_{k} e_{i} \tilde{\Delta}(W), \operatorname{dim}_{k} e_{i} \Lambda_{i l}(W), \operatorname{dim}_{k} e_{i} \Delta(W), \operatorname{dim}_{k} e_{i} W$ resp., if $\operatorname{type}(W)=\left(m_{1}, m_{2}, m_{3} ; r ; s_{1}, s_{2}, s_{3} ; t\right)$. The sufficiency of these 18 inequalities is a rather laborious checking which we will omit.

Remark. If $k$ is the field $\mathbf{F}_{2}$, the poset $\mathscr{P}_{n_{1}, n_{2}, n_{3}}$ is (isomorphic to) a refinement (see [6] for terminology) of the quotient poset $\left[\begin{array}{l}V \\ *\end{array}\right]_{k} / \mathrm{GL}_{A}(V)$. We still have the one-to-one correspondence between $\left[\begin{array}{l}V \\ *\end{array}\right]_{k} / \mathrm{GL}_{A}(V)$ and $\mathscr{P}_{n_{1}, n_{2}, n_{3}}$ as sets and the 18 inequalities are still necessary conditions for the order relation in the quotient poset; but they are not sufficient. In particular, in $\mathscr{P}_{n_{1}, n_{2}, n_{3}}$ we have $T \leq R$; but it is easily checked that the corresponding relation is not true in $\left[\begin{array}{l}V \\ *\end{array}\right]_{k} / \mathrm{GL}_{A}(V)$ : there is no subspace of $V$ of type $R$ containing a subspace of type $T$. One thing that does not change, however, is the size of the levels of the quotient poset, which corresponds to the size of some double coset spaces as we saw in $\S 3$.

Next follows additional information about the poset $\mathscr{P}_{n_{1}, n_{2}, n_{3}}$. It is ranked and Peck, of height $n_{1}+n_{2}+n_{3}$, with minimum element 0 , maximum element $M_{1}^{n_{1}} M_{2}^{n_{2}} M_{3}^{n_{3}}$, and the rank of an 8-tuple $\left(m_{1}, m_{2}, m_{3} ; r ; s_{1}, s_{2}, s_{3} ; t\right)$ equal to $m_{1}+m_{2}+m_{3}+2 r+s_{1}+s_{2}+s_{3}+t$. It is not a lattice: the two elements $M_{1} S_{1}$ and $R$ of rank 2 both cover the two elements $S_{1}$ and $T$ of rank 1 . If 
$\mathscr{P}_{n_{1}, n_{2}, n_{3}}^{(d)}$ denotes the $d$ th level of the poset and

$$
\begin{aligned}
& b_{d}=\mid\left\{\left(m_{1}, m_{2}, m_{3} ; r ; s_{1}, s_{2}, s_{3} ; t\right) \in \mathbb{N}^{8} \mid m_{1}+m_{2}+m_{3}+2 r\right. \\
& \left.+s_{1}+s_{2}+s_{3}+t=d\right\} \mid \\
& =\frac{1}{80640}\left(8 d^{7}+252 d^{6}+3248 d^{5}+22050 d^{4}+84392 d^{3}\right. \\
& \left.+179928 d^{2}+194592 d+80325\right)+\frac{1}{256}(-1)^{d} \\
& =\left\{\begin{array}{l}
\frac{1}{2520}(p+1)(p+2)(p+3)(p+4)\left(32 p^{3}+184 p^{2}+288 p+105\right) \\
\quad \text { for } d=2 p, \\
\frac{1}{2520} p(p+1)(p+2)(p+3)\left(32 p^{3}+200 p^{2}+352 p+151\right) \\
\text { for } d=2 p-1,
\end{array}\right.
\end{aligned}
$$

then we have the obvious bound $\left|\mathscr{P}_{n_{1}, n_{2}, n_{3}}^{(d)}\right| \leq b_{d}$, with equality when $d \leq$ $\min \left(n_{1}, n_{2}, n_{3}\right)$. (If $d \geq \frac{1}{2}\left(n_{1}+n_{2}+n_{3}\right)$, use the rank-symmetry of the poset to obtain a better bound.) We have the generating function

$$
\begin{aligned}
& \sum_{n_{1}, n_{2}, n_{3}, d \geq 0}\left|\mathscr{P}_{n_{1}, n_{2}, n_{3}}^{(d)}\right| x_{1}^{n_{1}} x_{2}^{n_{2}} x_{3}^{n_{3}} q^{d} \\
& =\frac{1}{\left(x_{1} ; q\right)_{2}\left(x_{2} ; q\right)_{2}\left(x_{3} ; q\right)_{2}\left(1-x_{1} x_{2} q\right)\left(1-x_{1} x_{3} q\right)\left(1-x_{2} x_{3} q\right)\left(x_{1} x_{2} x_{3} q ; q\right)_{2}},
\end{aligned}
$$

where we have used the standard abbreviation

$$
(a ; q)_{i}= \begin{cases}1 & \text { if } i=0, \\ (1-a)(1-a q) \cdots\left(1-a q^{i-1}\right) & \text { if } i=1,2, \ldots .\end{cases}
$$

The special case $q=1$ gives

$$
\begin{aligned}
& \sum_{n_{1}, n_{2}, n_{3} \geq 0}\left|\mathscr{P}_{n_{1}, n_{2}, n_{3}}\right| x_{1}^{n_{1}} x_{2}^{n_{2}} x_{3}^{n_{3}} \\
& \quad=\frac{1}{\left(1-x_{1}\right)^{2}\left(1-x_{2}\right)^{2}\left(1-x_{3}\right)^{2}\left(1-x_{1} x_{2}\right)\left(1-x_{1} x_{3}\right)\left(1-x_{2} x_{3}\right)\left(1-x_{1} x_{2} x_{3}\right)^{2}} .
\end{aligned}
$$

We now briefly sketch how to obtain the cardinality of $\mathscr{P}_{n, n, n}$, corresponding to the case $V$ free of rank $n$, from (2). Representing the RHS of (2) by power series, we have

$$
\left|\mathscr{P}_{n, n, n}\right|=\sum_{p=0}^{n}(n-p+1) f(p),
$$

where $f(p)$ is the coefficient of $x_{1}^{p} x_{2}^{p} x_{3}^{p}$ in

$$
\sum_{\substack{a_{1}, a_{2}, a_{3}, b_{1}, b_{2}, b_{3} \geq 0}}\left(a_{1}+1\right)\left(a_{2}+1\right)\left(a_{3}+1\right) x_{1}^{a_{1}+b_{2}+b_{3}} x_{2}^{a_{2}+b_{1}+b_{3}} x_{3}^{a_{3}+b_{1}+b_{2}} .
$$

After a change of variables $c_{1}=b_{2}+b_{3}, c_{2}=b_{1}+b_{3}, c_{3}=b_{1}+b_{2}$, one sees that

$$
f(p)=\sum_{(*)}\left(a_{1}+1\right)\left(a_{2}+1\right)\left(a_{3}+1\right),
$$


where the conditions on the parameters are

$$
\left\{\begin{array}{l}
0 \leq a_{1}, a_{2}, a_{3} \leq p \\
a_{1}+a_{2}+a_{3} \equiv p \quad(\bmod 2), \\
a_{i}+a_{j} \leq a_{l}+p \quad(\{i, j, l\} \text { permutation of }\{1,2,3\}) .
\end{array}\right.
$$

Using symmetry and inclusion-exclusion, we may write

$$
f(p)=3 I_{1}-3 I_{2}+I_{3}
$$

with

$$
I_{1}=\sum_{\substack{a_{3} \leq a_{1}, a_{2} \\(*)}} \cdots, \quad I_{2}=\sum_{\substack{a_{1}=a_{2} \leq a_{3} \\(*)}} \ldots, \quad I_{3}=\sum_{\substack{a_{1}=a_{2}=a_{3} \\(*)}} \cdots .
$$

A case-by-case computation of $I_{1}, I_{2}, I_{3}$ according to the parity of $p, a_{1}, a_{2}$, $a_{3}$ yields the following

\section{Proposition 5.8.}

$$
\begin{aligned}
\left|\mathscr{P}_{n, n, n}\right|= & \frac{1}{26880}(n+1)(n+3)^{2}(n+5)\left(11(n+3)^{4}-40(n+3)^{2}+64\right) \\
& + \begin{cases}0 & \text { for } n \text { odd }, \\
\frac{105}{26880} & \text { for } n \text { even. }\end{cases}
\end{aligned}
$$

In particular, $\left|\mathscr{P}_{n, n, n}\right| \sim \frac{11}{26880} n^{8}$ as $n \rightarrow \infty$.

\section{THE CASE $A=F \times k$}

In this section $A=F \times k$, where $F / k$ is a quadratic field extension, and $V$ is an $A$-module of finite bidimension $\left(n_{1}, n_{2}\right)$. We fix an element $\eta \in F$ such that $F=k(\eta)$.

We first collect the following facts (see for example [4]) about subspaces of a vector space over a quadratic field extension, which we will freely use below.

Theorem 6.1. Let $k, F$, and $\eta$ be as above. Let $U$ be a finite-dimensional vector space over $F$ and let $W \in\left[\begin{array}{c}U \\ *\end{array}\right]_{k}$.

(a) $\operatorname{comp}_{F} W=W \cap \eta W$; for $x \in W$, we have $x \in \operatorname{comp}_{F} W \Leftrightarrow \eta x \in W$.

(b) If $\operatorname{comp}_{F} W=0$, then $F W=W \oplus \eta W, \operatorname{dim}_{F} F W=\operatorname{dim}_{k} W$, and any $k$-independent vectors $v_{1}, \ldots, v_{r}$ in $W$ are $F$-independent.

(c) Suppose $W=W_{1} \oplus Y$ for some $k$-subspaces $W_{1}, Y$ with comp com $_{F} W W_{1}$. Then $\operatorname{comp}_{F} Y=0, W=W_{1} \oplus_{F} Y$, and $\operatorname{dim}_{k} Y=\operatorname{dim}_{F}\left(F W / F W_{1}\right)$.

(d) Let $W_{1} \in\left[\begin{array}{c}W \\ *\end{array}\right]_{k}$ satisfy $W_{1}=W \cap F W_{1}$ (equivalently, $W_{1}=W \cap U_{1}$ for some $\left.U_{1} \in\left[\begin{array}{c}U \\ *\end{array}\right]_{F}\right)$. Then there exists a subspace $Z \in\left[\begin{array}{c}W \\ *\end{array}\right]_{k}$ such that $W=W_{1} \oplus_{F} Z$.

For a $k$-subspace $W$ of $V$, let $\Lambda_{i}(W)=W \cap e_{i} V(i=1,2)$ and define the type of $W$ to be the 6-tuple

$$
\begin{array}{r}
\operatorname{type}(W)=\left(\operatorname{dim}_{F} \operatorname{comp}_{F} \Lambda_{1}(W), \operatorname{dim}_{k} \Lambda_{2}(W) ; \operatorname{dim}_{F}\left(U / \operatorname{comp}_{F} \Lambda_{1}(W)\right),\right. \\
\operatorname{dim}_{F}\left(F \Lambda_{1}(W) / U\right), \operatorname{dim}_{F}\left(\left(\operatorname{comp}_{F} e_{1} W\right) / U\right), \\
\left.\operatorname{dim}_{F}\left(F e_{1} W /\left(F \Lambda_{1}(W)+\operatorname{comp}_{F} e_{1} W\right)\right)\right)
\end{array}
$$

with $U=F \Lambda_{1}(W) \cap \operatorname{comp}_{F} e_{1} W$. The parameters in type $(W)$ are invariant 
under the action of $\mathrm{GL}_{A}(V)$ on $\left[\begin{array}{l}V \\ *\end{array}\right]_{k}$ and will form a complete set of invariants for this action.

Theorem 6.2. Let $W \in\left[\begin{array}{l}V \\ { }_{*}\end{array}\right]_{k}$ with type $(W)=\left(m_{1}, m_{2} ; b, c, e, f\right)$. There exist $F$-independent vectors $v_{1}^{(1)}, \ldots, v_{m_{1}}^{(1)} ; x_{1}^{(1)}, \ldots, x_{b}^{(1)} ; y_{1}, \ldots, y_{c} ; z_{1}^{(1)}, \ldots$, $z_{e}^{(1)} ; u_{1}^{(1)}, \ldots, u_{f}^{(1)}$ in $e_{1} V$ and $k$-independent vectors $v_{1}^{(2)}, \ldots, v_{m_{2}}^{(2)}$; $x_{1}^{(2)}, \ldots, x_{b}^{(2)} ; z_{1}^{(2)}, \ldots, z_{2 e}^{(2)} ; u_{1}^{(2)}, \ldots, u_{f}^{(2)}$ in $e_{2} V$ such that $W$ admits the direct sum decomposition over $A$

$$
W=\operatorname{comp}_{F} \Lambda_{1}(W) \oplus_{A} \Lambda_{2}(W) \oplus_{A} X \oplus_{A} Y \oplus_{A} Z \oplus_{A} U
$$

with

$$
\begin{aligned}
& \operatorname{comp}_{F} \Lambda_{1}(W)=\left\langle v_{1}^{(1)}, \ldots, v_{m_{1}}^{(1)}\right\rangle_{F}, \quad \Lambda_{2}(W)=\left\langle v_{1}^{(2)}, \ldots, v_{m_{2}}^{(2)}\right\rangle_{k}, \\
& X=\bigoplus_{i=1}^{b}\left\langle x_{i}^{(1)}, \eta x_{i}^{(1)}+x_{i}^{(2)}\right\rangle_{k}, \quad Y=\left\langle y_{1}, \ldots, y_{c}\right\rangle_{k}, \\
& Z=\bigoplus_{i=1}^{e}\left\langle z_{i}^{(1)}+z_{2 i-1}^{(2)}, \eta z_{i}^{(1)}+z_{2 i}^{(2)}\right\rangle_{k}, \quad U=\bigoplus_{i=1}^{f}\left\langle u_{i}^{(1)}+u_{i}^{(2)}\right\rangle_{k} .
\end{aligned}
$$

Moreover, if $\pi$ is the map $v \mapsto e_{1} v$ from $W$ to $e_{1} V$, we have

$$
\begin{gathered}
\Lambda_{1}(W)=\operatorname{comp}_{F} \Lambda_{1}(W) \oplus_{F}\left\langle x_{1}^{(1)}, \ldots, x_{b}^{(1)}\right\rangle_{k} \oplus_{F} Y \\
F \Lambda_{1}(W)=\operatorname{comp}_{F} \Lambda_{1}(W) \oplus_{F} e_{1} X \oplus_{F} F Y \\
\operatorname{comp}_{F} e_{1} W=\operatorname{comp}_{F} \Lambda_{1}(W) \oplus_{F} e_{1} X \oplus_{F} e_{1} Z
\end{gathered}
$$

and

$$
\begin{aligned}
& \operatorname{comp}_{A} W=\operatorname{comp}_{F} \Lambda_{1}(W) \oplus_{A} \Lambda_{2}(W), \\
& \pi^{-1}\left(F \Lambda_{1}(W) \cap \operatorname{comp}_{F} e_{1} W\right)=\operatorname{comp}_{F} \Lambda_{1}(W) \oplus_{A} \Lambda_{2}(W) \oplus_{A} X, \\
& \pi^{-1}\left(F \Lambda_{1}(W)\right)=\operatorname{comp}_{F} \Lambda_{1}(W) \oplus_{A} \Lambda_{2}(W) \oplus_{A} X \oplus_{A} Y, \\
& \pi^{-1}\left(\operatorname{comp}_{F} e_{1} W\right)=\operatorname{comp}_{F} \Lambda_{1}(W) \oplus_{A} \Lambda_{2}(W) \oplus_{A} X \oplus_{A} Z, \\
& \pi^{-1}\left(F \Lambda_{1}(W)+\operatorname{comp}_{F} e_{1} W\right)=\operatorname{comp}_{F} \Lambda_{1}(W) \oplus_{A} \Lambda_{2}(W) \oplus_{A} X \oplus_{A} Y \oplus_{A} Z .
\end{aligned}
$$

Proof. The proof will involve a series of lemmas. By Proposition 2.5,

$$
\operatorname{comp}_{A} W=\operatorname{comp}_{F} \Lambda_{1}(W) \oplus_{A} \Lambda_{2}(W) .
$$

Choose a basis $\left\{v_{1}^{(1)}, \ldots, v_{m_{1}}^{(1)}\right\}$ of $\operatorname{comp}_{F} \Lambda_{1}(W)$ over $F$ and a basis $\left\{v_{1}^{(2)}\right.$, $\left.\ldots, v_{m_{2}}^{(2)}\right\}$ of $\Lambda_{2}(W)$ over $k$. Using Proposition 2.4, we are now allowed to assume that $\operatorname{comp}_{A} W=0$, as in the proof of Theorem 5.1; so $\operatorname{comp}_{F} \Lambda_{1}(W)=$ 0 and $\Lambda_{2}(W)=0$.

Let $\varphi$ denote the map $v \mapsto e_{1} v$ from $W$ onto $e_{1} W$, i.e., $\varphi$ is the same map as $\pi$ but with restricted codomain. Note that $\varphi$ is a $k$-linear isomorphism (because its kernel equals $W \cap e_{2} V=\Lambda_{2}(W)=0$ ) with $\Lambda_{1}(W)$ as a set of fixed points. 
Put $\tilde{X}=F \Lambda_{1}(W) \cap \operatorname{comp}_{F} e_{1} W \in\left[\begin{array}{c}e_{1} W \\ b\end{array}\right]_{F}$ and let $X=\varphi^{-1}(\widetilde{X}) \in\left[\begin{array}{c}W \\ 2 b\end{array}\right]_{k}$. Choose a basis $\left\{x_{1}^{(1)}, \ldots, x_{b}^{(1)}\right\}$ of $\widetilde{X}$ over $F$ consisting of vectors of $\Lambda_{1}(W)$. For $i=1, \ldots, b$, we have $\eta x_{i}^{(1)} \in e_{1} W$; take $x_{i}^{(2)}=e_{2} \varphi^{-1}\left(\eta x_{i}^{(1)}\right)$, so that $\varphi^{-1}\left(\eta x_{i}^{(1)}\right)=\eta x_{i}^{(1)}+x_{i}^{(2)}$ and $X=\bigoplus_{i=1}^{b}\left\langle x_{i}^{(1)}, \eta x_{i}^{(1)}+x_{i}^{(2)}\right\rangle_{k}$. We have $e_{1} X=\tilde{X}$ and $\left\langle x_{1}^{(2)}, \ldots, x_{b}^{(2)}\right\rangle_{k}=e_{2} \varphi^{-1}(\tilde{X})$.

Lemma 6.3. (i) The vectors $x_{1}^{(2)}, \ldots, x_{b}^{(2)}$ are $k$-independent.

(ii) $\Lambda_{1}(W) \cap \tilde{X}=\left\langle x_{1}^{(1)}, \ldots, x_{b}^{(1)}\right\rangle_{k}=\Lambda_{1}(W) \cap X$.

(iii) $\widetilde{X}=F\left(\Lambda_{1}(W) \cap \tilde{X}\right)$.

Proof. (i) It suffices to show that the $k$-linear map $x \mapsto e_{2} \varphi^{-1}(\eta x)$ from $\Lambda_{1}(W) \cap \tilde{X}$ to $e_{2} V$ is injective. Suppose $x \in \Lambda_{1}(W) \cap \tilde{X}$ and $e_{2} \varphi^{-1}(\eta x)=0$. Then $\varphi^{-1}(\eta x) \in e_{1} V \cap W=\Lambda_{1}(W)$, which implies $\eta x \in \Lambda_{1}(W)$ and $x \in$ $\operatorname{comp}_{F} \Lambda_{1}(W)=0$.

(ii) and (iii) are now easy to derive.

Note that $\tilde{X}=\operatorname{comp}_{F}\left(F \Lambda_{1}(W) \cap e_{1} W\right)$ by Lemma 2.3. So by Proposition 2.4 there exists a $k$-subspace $Y$ of $F \Lambda_{1}(W) \cap e_{1} W$ such that

$$
F \Lambda_{1}(W) \cap e_{1} W=\tilde{X} \oplus_{F} Y .
$$

Lemma 6.4. (i) $\operatorname{comp}_{F} Y=0$.

(ii) $\Lambda_{1}(W)=\left(\Lambda_{1}(W) \cap \tilde{X}\right) \oplus_{F} Y$.

(iii) $F \Lambda_{1}(W)=\widetilde{X} \oplus_{F} F Y$.

(iv) $\operatorname{dim}_{k} Y=c$.

Proof. (i) is clear.

To prove (ii) we first show that $Y \leq \Lambda_{1}(W)$. Let $0 \neq y \in Y$. We can write $y=\lambda x$ for some $\lambda \in F$ and $x \in \Lambda_{1}(W)$. We claim that $\lambda \in k$. Otherwise, the fact that $x \in e_{1} W$ and $\lambda x=y \in e_{1} W$ implies that $x \in$ $\operatorname{comp}_{F} e_{1} W \cap F \Lambda_{1}(W)=\tilde{X}$, and hence $y \in \tilde{X}$, which is a contradiction. The claim now follows from the inclusion $\Lambda_{1}(W) \leq F \Lambda_{1}(W) \cap e_{1} W=\tilde{X} \oplus_{F} Y$.

(iii) follows from (ii) and Lemma 6.3(iii).

To prove (iv), use (i), (iii), and the definition of type( $W$ ), to get $\operatorname{dim}_{k} Y=$ $\operatorname{dim}_{F} F Y=\operatorname{dim}_{F}\left(F \Lambda_{1}(W) / \widetilde{X}\right)=c$.

Choose a basis $\left\{y_{1}, \ldots, y_{c}\right\}$ of $Y$ over $k$. Since $\operatorname{comp}_{F} Y=0$, the vectors $y_{1}, \ldots, y_{c}$ are actually independent over $F$.

Pick an $F$-subspace $\tilde{Z}$ of $\operatorname{comp}_{F} e_{1} W$ such that

$$
\operatorname{comp}_{F} e_{1} W=\tilde{X} \oplus_{F} \tilde{Z} \text {. }
$$

Necessarily $\operatorname{dim}_{F} \tilde{Z}=e$. Let $Z=\varphi^{-1}(\tilde{Z}) \in\left[\begin{array}{c}W \\ 2 e\end{array}\right]_{k}$. Choose a basis $\left\{z_{1}^{(1)}, \ldots\right.$, $\left.z_{e}^{(1)}\right\}$ of $\tilde{Z}$ over $F$. For $i=1, \ldots, e$, put $z_{2 i-1}^{(2)}=e_{2} \varphi^{-1}\left(z_{i}^{(1)}\right)$ and $z_{2 i}^{(2)}=$ $e_{2} \varphi^{-1}\left(\eta z_{i}^{(1)}\right)$ so that $Z=\bigoplus_{i=1}^{e}\left\langle z_{i}^{(1)}+z_{2 i-1}^{(2)}, \eta z_{i}^{(1)}+z_{2 i}^{(2)}\right\rangle_{k}$. We have $e_{1} Z=\tilde{Z}$ and $\left\langle z_{1}^{(2)}, \ldots, z_{2 e}^{(2)}\right\rangle_{k}=e_{2} \varphi^{-1}(\widetilde{Z})$. 
Lemma 6.5. (i) $\tilde{Z} \cap F \Lambda_{1}(W)=0$.

(ii) The sum $\tilde{X} \oplus_{F} Y \oplus_{F} \widetilde{Z}$ is direct over $F$.

(iii) The vectors $z_{1}^{(2)}, \ldots, z_{2 e}^{(2)}$ are $k$-independent in $e_{2} V$.

(iv) $e_{1} W \cap\left(F \Lambda_{1}(W)+\operatorname{comp}_{F} e_{1} W\right)=\tilde{X} \oplus_{F} Y \oplus_{F} \tilde{Z}$.

Proof. (i) and (ii) are easy to derive from Lemma 6.4(iii). To prove (iii) it suffices to show that the $k$-linear map $z \mapsto e_{2} \varphi^{-1}(z)$ from $\widetilde{Z}$ to $e_{2} V$ is injective. Suppose $z \in \widetilde{Z}$ and $e_{2} \varphi^{-1}(z)=0$. Then $z \in \Lambda_{1}(W) \cap \tilde{Z}=0$ by (i). For (iv) it is easy to see that $e_{1} W \cap F Y=Y$. The result then follows from the facts that $F \Lambda_{1}(W)+\operatorname{comp}_{F} e_{1} W=\tilde{X}+F Y+\widetilde{Z}$ and $\tilde{X}+\tilde{Z} \leq e_{1} W$.

Applying Theorem 6.1(d) to the situation of Lemma 6.5(iv), we can find a $k$-subspace $\tilde{U}$ of $e_{1} W$ such that

$$
e_{1} W=\tilde{X} \oplus_{F} Y \oplus_{F} \tilde{Z} \oplus_{F} \tilde{U}
$$

We have $\operatorname{comp}_{F} \tilde{U}=0$ and

$$
\begin{aligned}
F e_{1} W & =\tilde{X} \oplus F Y \oplus \widetilde{Z} \oplus F \widetilde{U} \\
& =\left(F \Lambda_{1}(W)+\operatorname{comp}_{F} e_{1} W\right) \oplus F \widetilde{U}
\end{aligned}
$$

so $\operatorname{dim}_{k} \tilde{U}=\operatorname{dim}_{F} F \tilde{U}=e$. Let $U=\varphi^{-1}(\tilde{U}) \in\left[\begin{array}{c}W \\ f\end{array}\right]_{k}$. Choose a basis $\left\{u^{(1)}, \ldots, u_{f}^{(1)}\right\}$ of $\tilde{U}$ over $k$; the vectors $u_{1}^{(1)}, \ldots, u_{f}^{(1)}$ are $F$-independent since $\operatorname{comp}_{F} \tilde{U}=0$. For $i=1, \ldots, f$, put $u_{i}^{(2)}=e_{2} \varphi^{-1}\left(u_{i}^{(1)}\right)$ so that $U=$ $\bigoplus_{i=1}^{f}\left\langle u_{i}^{(1)}+u_{i}^{(2)}\right\rangle_{k}$. We have $e_{1} U=\widetilde{U}$ and $\left\langle u_{1}^{(2)}, \ldots, u_{f}^{(2)}\right\rangle_{k}=e_{2} \varphi^{-1}(\widetilde{U})$. Note also that the vectors $x_{1}^{(1)}, \ldots, x_{b}^{(1)} ; y_{1}, \ldots, y_{c} ; z_{1}^{(1)} \ldots, z_{e}^{(1)} ; u_{1}^{(1)}, \ldots, u_{f}^{(1)}$ are all independent over $F$ because the sum in (1) is direct over $F$.

Lemma 6.6. (i) The vectors $u_{1}^{(2)}, \ldots, u_{f}^{(2)}$ are $k$-independent in $e_{2} V$.

(ii) We have a direct sum $e_{2} \varphi^{-1}(\tilde{X}) \oplus e_{2} \varphi^{-1}(\tilde{Z}) \oplus e_{2} \varphi^{-1}(\tilde{U})$.

(iii) The vectors $x_{1}^{(2)}, \ldots, x_{b}^{(2)} ; z_{1}^{(2)}, \ldots, z_{2 e}^{(2)} ; u_{1}^{(2)}, \ldots, u_{f}^{(2)}$ are all $k$ independent.

(iv) $W=X \oplus_{A} Y \oplus_{A} Z \oplus_{A} U$.

Proof. (i) is proved exactly like Lemma 6.5(iii).

(ii) Suppose $e_{2} \varphi^{-1}(x)+e_{2} \varphi^{-1}(z)+e_{2} \varphi^{-1}(u)=0$ with $x \in \tilde{X}, z \in \tilde{Z}, u \in \tilde{U}$. Then $e_{2} \varphi^{-1}(x+z+u)=0$ implies $x+z+u \in \Lambda_{1}(W)$. Since $\widetilde{X} \leq F \Lambda_{1}(W)$, we have $z+u \in F \Lambda_{1}(W) \cap(\tilde{Z} \oplus \tilde{U})=0$ and $e_{2} \varphi^{-1}(x)=0$. Since $\tilde{Z} \cap \tilde{U}=0$, we also have $z=u=0$ and $e_{2} \varphi^{-1}(z)=e_{2} \varphi^{-1}(u)=0$.

(iii) is a consequence of (ii).

(iv) We have $W=\varphi^{-1}\left(e_{1} W\right)=X \oplus Y \oplus Z \oplus U$. Formula (1) and (ii) combined with Proposition 2.6 show that this sum is direct over $A$.

The equalities at the end of the statement of the theorem are trivial to check. The proof of Theorem 6.2 is now complete. 
Remark. If type $(W)=\left(m_{1}, m_{2} ; b, c, e, f\right)$, then

$$
\begin{aligned}
& \operatorname{dim}_{k} \Lambda_{1}(W)=2 m_{1}+b+c, \\
& \operatorname{dim}_{F} F \Lambda_{1}(W)=m_{1}+b+c, \\
& \operatorname{dim}_{F} \operatorname{comp}_{F} e_{1} W=m_{1}+b+e, \\
& \operatorname{dim}_{k} e_{1} W=2 m_{1}+2 b+c+2 e+f, \\
& \operatorname{dim}_{F} F e_{1} W=m_{1}+b+c+e+f, \\
& \operatorname{dim}_{k} e_{2} W=m_{2}+b+2 e+f, \\
& \operatorname{dim}_{k} W=2 m_{1}+m_{2}+2 b+c+2 e+f, \\
& \operatorname{dim}_{k} A W=2 m_{1}+m_{2}+3 b+2 c+4 e+3 f .
\end{aligned}
$$

We see that a 6-tuple $\left(m_{1}, m_{2} ; b, c, e, f\right) \in \mathbb{N}^{6}$ is the type of some $k$ subspace of $V$ iff

$$
m_{1}+b+c+e+f \leq n_{1}, \quad m_{2}+b+2 e+f \leq n_{2} .
$$

The next two results are the expected analogues of Theorems 5.6 and 5.7.

Theorem 6.7. Two $k$-subspaces of $V$ are in the same orbit for $\mathrm{GL}_{A}(V)$ if and only if they have the same type.

Theorem 6.8. Let $A$ be the algebra $F \times k$ with $F / k$ quadratic extension and let $V$ be an A-module of finite bidimension $\left(n_{1}, n_{2}\right)$. The quotient poset $\left[\begin{array}{l}V \\ *\end{array}\right]_{k} / \mathrm{GL}_{A}(V)$ is finite and independent of the fields $k$ and $F$. It is isomorphic to the poset

$$
\begin{aligned}
\mathscr{P}_{n_{1}, n_{2}}=\left\{\left(m_{1}, m_{2} ; b, c, e, f\right) \in \mathbb{N}^{6} \mid m_{1}+b+c+e+f \leq n_{1},\right. \\
\\
\left.\quad m_{2}+b+2 e+f \leq n_{2}\right\},
\end{aligned}
$$

with the order relation

$$
\left(m_{1}, m_{2} ; b, c, e, f\right) \leq\left(m_{1}^{\prime}, m_{2}^{\prime} ; b^{\prime}, c^{\prime}, e^{\prime}, f^{\prime}\right)
$$

given by the following ten irredundant conditions:

$$
\begin{aligned}
m_{1} & \leq m_{1}^{\prime}, \\
m_{1}+b & \leq m_{1}^{\prime}+b^{\prime}, \\
m_{1}+b+c & \leq m_{1}^{\prime}+b^{\prime}+c^{\prime}, \\
m_{1}+b+e & \leq m_{1}^{\prime}+b^{\prime}+e^{\prime}, \\
m_{1}+b+c+e & \leq m_{1}^{\prime}+b^{\prime}+c^{\prime}+e^{\prime}, \\
m_{1}+b+c+e+f & \leq m_{1}^{\prime}+b^{\prime}+c^{\prime}+e^{\prime}+f^{\prime}, \\
m_{2} & \leq m_{2}^{\prime}, \\
m_{2}+b & \leq m_{2}^{\prime}+b^{\prime}, \\
m_{2}+b+2 e & \leq m_{2}^{\prime}+b^{\prime}+2 e^{\prime}, \\
m_{2}+b+2 e+f & \leq m_{2}^{\prime}+b^{\prime}+2 e^{\prime}+f^{\prime} .
\end{aligned}
$$


Note that the combinations of parameters used to define the order relation in $\mathscr{P}_{n_{1}, n_{2}}$ are obtained by taking $\operatorname{dim}_{F} F e_{1} C$ and $\operatorname{dim}_{k} e_{2} C$ for each of the last five subspaces $C$ appearing in the statement of Theorem 6.2, as well as $W$.

We conclude by stating additional information about the poset $\mathscr{P}_{n_{1}, n_{2}}$. It is ranked and Peck, of height $2 n_{1}+n_{2}$, with minimum element $(0,0 ; 0,0,0,0)$, and maximum element $\left(n_{1}, n_{2} ; 0,0,0,0\right)$; the rank of a 6-tuple $\left(m_{1}, m_{2}\right.$; $b, c, e, f)$ equals $2 m_{1}+m_{2}+2 b+c+2 e+f$. The poset is not a lattice: the two elements $(0,0 ; 1,0,0,0)$ and $(0,1 ; 0,1,0,0)$ of rank 2 both cover the two elements $(0,0 ; 0,0,0,1)$ and $(0,0 ; 0,1,0,0)$ of rank 1 . Let $\mathscr{P}_{n_{1}, n_{2}}^{(d)}$ denote the $d$ th level of the poset and let

$$
\begin{aligned}
b_{d}= & \left|\left\{\left(m_{1}, m_{2} ; b, c, e, f\right) \in \mathbb{N}^{6} \mid 2 m_{1}+m_{2}+2 b+c+2 e+f=d\right\}\right| \\
= & \frac{1}{3840}\left(4 d^{5}+90 d^{4}+760 d^{3}+2970 d^{2}+5266 d\right. \\
& \left.\quad+3285+(-1)^{d}\left(30 d^{2}+270 d+555\right)\right) \\
= & \begin{cases}\frac{1}{120}(p+1)_{4}(4 p+5) & \text { for } d=2 p, \\
\frac{1}{120}(p)_{4}(4 p+11) & \text { for } d=2 p-1,\end{cases}
\end{aligned}
$$

where we have used the standard abbreviation for rising factorials

$$
(a)_{i}= \begin{cases}1 & \text { if } i=0, \\ a(a+1) \cdots(a+i-1) & \text { if } i=1,2, \ldots\end{cases}
$$

Then

$$
\left|\mathscr{P}_{n_{1}, n_{2}}^{(d)}\right| \leq b_{d},
$$

with equality when $d \leq \min \left(n_{1}, n_{2}\right)$. We have the generating function

$$
\sum_{n_{1}, n_{2}, d \geq 0}\left|\mathscr{P}_{n_{1}, n_{2}}^{(d)}\right| x_{1}^{n_{1}} x_{2}^{n_{2}} q^{d}=\frac{1}{\left(x_{1} ; q\right)_{3}\left(x_{2} ; q\right)_{2}\left(x_{1} x_{2} q ; q\right)_{2}\left(1-x_{1} x_{2}^{2} q^{2}\right)} .
$$

The special case $q=1$ gives

$$
\sum_{n_{1}, n_{2} \geq 0}\left|\mathscr{P}_{n_{1}, n_{2}}\right| x_{1}^{n_{1}} x_{2}^{n_{2}}=\frac{1}{\left(1-x_{1}\right)^{3}\left(1-x_{2}\right)^{2}\left(1-x_{1} x_{2}\right)^{2}\left(1-x_{1} x_{2}^{2}\right)} .
$$

From this last generating function, one obtains the cardinality of $\mathscr{P}_{n, n}$ corresponding to the case $V$ free of rank $n$ :

$$
\left|\mathscr{P}_{n, n}\right|=\left\{\begin{array}{rr}
\frac{1}{360}(p+1)(p+2)\left(68 p^{4}+408 p^{3}+835 p^{2}+669 p+180\right) \\
\text { for } n=2 p \\
\frac{1}{90} p(p+1)^{2}(p+2)\left(17 p^{2}+34 p+9\right) & \text { for } n=2 p-1
\end{array}\right.
$$

In particular, $\left|\mathscr{P}_{n, n}\right| \sim \frac{17}{5760} n^{6}$ as $n \rightarrow \infty$. Also,

$$
\left|\mathscr{P}_{n_{1}, n_{2}}\right|=\frac{1}{360}\left(n_{1}+1\right)_{5}\left(3 n_{2}-2 n_{1}+3\right) \quad \text { if } n_{2} \geq 2 n_{1} .
$$

We could also give formulas for the cases $n_{1} \leq n_{2} \leq 2 n_{1}$ and $n_{2} \leq n_{1}$, but the results do not factor nicely. 


\section{REFERENCES}

1. S. Gelbart, I. Piatetski-Shapiro, and S. Rallis, Explicit constructions of automorphic Lfunctions, Lecture Notes in Math., vol. 1254, Springer-Verlag, New York, 1987.

2. I. Gessel and D. Stanton, Strange evaluations of hypergeometric series, Siam J. Math. Anal. 13 (1982), 295-308.

3. D. S. Kim and P. Rabau, Field extensions and isotropic subspaces in symplectic geometry, Geom. Dedicata 34 (1990), 281-293.

4. P. Rabau, Action on Grassmannians associated with a field extension, Trans. Amer. Math. Soc. 326 (1991), 127-155.

5. R. Stanley, Quotients of Peck posets, Order 1 (1984), 29-34.

6. __ Enumerative combinatorics, vol. 1, Wadsworth, Monterey, Calif., 1986.

Department of Mathematics, Seoul Woman's University, Seoul, 139-744, Korea

Department of Mathematics, The Ohio State University, Columbus, Ohio 43210 